\title{
Gestos comunicativos y contextos interpersonales: un estudio con niños de 10 a 16 meses.
}

\section{Español, Silvia y Angel Riviére.}

Cita:

Español, Silvia y Angel Riviére (2000). Gestos comunicativos y contextos interpersonales: un estudio con niños de 10 a 16 meses. eISSN: 1579-3699 - Estudios de Psicología, 65, 225-245.

Dirección estable: https://www.aacademica.org/silvia.espanol/119 ARK: https://n2t.net/ark:/13683/pHOV/zmb 


\section{Estudios de Psicología}

\section{Gestos comunicativos y contextos interpersonales: un estudio con niños de 10 a 16 meses}

\section{Silvia Español \& Ángel Rivière}

To cite this article: Silvia Español \& Ángel Rivière (2000) Gestos comunicativos y contextos interpersonales: un estudio con niños de 10 a 16 meses, Estudios de Psicología, 21:65-66, 225-245

To link to this article: http://dx.doi.org/10.1174/021093900320380785

册 Published online: 23 Jan 2014.

Submit your article to this journal 전

Џ Article views: 42

Q View related articles ¿

4 Citing articles: 6 View citing articles ๘ 


\title{
Gestos comunicativos y contextos interpersonales: un estudio con niños de 10 a 16 meses
}

\author{
SILVIA ESPAÑOL Y ÁNGEL RIVIÈRE \\ Universidad Autónoma de Madrid
}

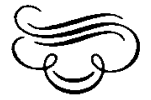

Resumen

En este trabajo se investiga la producción del gesto de señalar y otros gestos relacionados (gesto indicativo y «reaching») en 27 niños de 10, 13 y 16 meses de edad, en una condición comunicativa general orientada a favorecer la función declarativa del gesto en la que se variaron los grados de contacto interpersonal entre el niño y el adulto que se encuentra con él. Los grados de contacto interpersonal fueron alto, medio y bajo. El análisis de los datos se realizó en relación a la frecuencia de producción de los gestos y la dirección de la mirada del niño, en cada una de las tres situaciones y en cada uno de los tres grupos de edad. Los resultados muestran el alto grado de sensibilidad de los niños al contexto interpersonal en el que se encuentran, que se manifiesta tanto en la frecuencia de producción del gesto de señalar y del gesto indicativo como en el uso que hacen de la mirada. Indican, también, la básica condición social del gesto de señalar para sí mismo y su aparición simultánea en el desarrollo con el gesto de señalar con fines comunicativos. Sugieren, a su vez, una equivalencia funcional entre el gesto de señalar para sí mismo y el gesto indicativo.

Palabras claves: Comunicación preverbal, gestos, gesto de señalar.

\section{Communicative gestures and interpersonal contexts: A study with 10 to 16 months old infants}

\begin{abstract}
This study focuses on the production of the pointing gesture and other related gestures (indicative gesture and reaching) in a sample of 27 infants aged 10,13 and 16 months in a general communicative situation designed to arouse the declarative function of the gesture. The research was carried out under three experimental conditions of interpersonal contact between the child and the adult: high, medium and low level. The data were analysed according to the frequency of production of gestures and the direction of gaze of the child under the different experimental conditions in the three age groups. Results report a high level of sensitivity of the child towards the social context; this becomes evident not only in the frequency of production of the pointing and of the indicative gestures but as well in the use of the gaze. Results also higblight the basic social condition of the pointing for self and the simultaneous appearance in the development of the communicative pointing gesture. At the same time, the data suggest a functional equivalence between the pointing for self and the indicative gesture.
\end{abstract}

Keywords: Preverbal communication, gestures, pointing gesture.

Agradecimientos: Agradecemos a Ignacio Montero García Celay el asesoramiento metodológico. Esta investigación forma parte del Proyecto PB-97-0058 financiado por el Programa Sectorial de Promoción del Conocimiento de la Dirección General de Investigación Científica y Técnica (DGICYT) del Ministerio de Educación y Ciencia.

Correspondencia con los autores: Departamento de Psicología Básica. Facultad de Psicología. Universidad Autónoma de Madrid.Campus de Cantoblanco.28049 Madrid.E-mail: silvia.espannol@uam.es 


\section{INTRODUCCIÓN}

Desde el momento de su nacimiento, los niños se encuentran inmersos en un mundo semiótico, un mundo en el que sus primeros movimientos y expresiones son interpretados por las personas que lo rodean. De éste modo — dado que su conducta es significativa para los otros y que éstos actúan en función de sus interpretaciones - el bebé log ra regular la conductas de los otros, aunque él no lo sepa ni tenga la intención de hacerlo. Las acciones del bebé, durante sus primeros meses de vida, no son acciones que sean sígnicas desde su propia perspectiva mental pero sí son signos que tienen consecuencias en el adulto, de ahí que se distinga una fase perlocutiva (Bates 1976), previa a cualquier intención comunicativa, en el desarrollo de la comunicación humana. En momentos posteriores del desarrollo, el niño podrá a través del lenguaje regular su conducta y la de los otros. Entre el momento en que el niño interactúa con sus figuras de crianza y logra regular sus conductas, aunque no lo sepa ni lo pretenda, y el momento en que se comunica intencionalmente con las personas que lo rodean a través del lenguaje, se extiende un período, psicológicamente rico y complejo, en el que el niño empieza a emitir signos preverbales con la intención de que signifiquen algo para el otro, es decir comienza a generar sus primeros gestos comunicativos. Estas primeras pautas de relación intencionadamente comunicativas se manifiestan durante el último trimestre del primer año de vida del niño; sus primeros atisbos, hacia los nueve meses, inauguran una fase del desarrollo, a la que Bates (1976) denominó fase ilocutiva. Es éste un momento crucial en el desarrollo de las competencias semióticas del niño que implican que, de algún modo, el niño se torna consciente de estar produciendo una actividad que no ejerce efectos materiales sobre el mundo sino que tiene consecuencias mentales en sus compañeros de interacción. Este período —al que podemos caracterizar como aquél en el que el niño se hace consciente de que su propia actividad es una actividad sígnica dirigida a producir un significado en el otro- es el que nos ocupa en este trabajo.

Desde hace tres décadas el interés por la producción gestual infantil ha ido en aumento. Las explicaciones del origen y función de los gestos se han realizado desde perspectivas en las que confluyen dos grandes tradiciones psicológicas: la teoría piagetiana de la inteligencia sensoriomotora y las ideas vygotskianas acerca del origen de los procesos psicológicos superiores. A su vez, especialmente en la última década, se ha desarrollado un interés creciente acerca de las habilidades sociales implicadas en la producción y comprensión de gestos. Se han diferenciado varias funciones comunicativas en el uso de gestos; dos de ellas tienen una importancia esencial y han dado nombre a las llamadas pautas protoimperativas y protodeclarativas. (Bates, Camaioni y Volterra, 1975; Bates, 1976). Las pautas protoimperativas implican intentos de lograr cambios en el mundo a través de las personas. Las pautas protodeclarativas, tienen una intención más genuinamente social: parecen buscar compartir la atención y el interés por los estados del mundo. Así, un niño puede señalar un objeto al adulto con la intención de que éste se lo alcance, o puede señalarlo sin otra finalidad que compartir con el adulto el interés por el objeto. En los años 70 se consideró que estas habilidades comunicativas de los niños podían explicarse apelando a la inteligencia sensoriomotora; ellas implicaban la comprensión de una relación medios-fines en la que interviene un adulto al que el niño atribuye causalidad independiente como agente. El paralelismo entre el desarrollo del uso de instrumentos físicos y el desarrollo del uso de instrumentos semióticos llevó a postular el desarrollo de la causalidad del quinto estadio piagetiano como un requisito para la comunicación intencio- 
nal y a plantear la existencia de una correlación entre inteligencia sensoriomotora y comunicación intencional. Sin embargo, pocos años después Sarriá y Rivière (1991) señalaron la presencia de datos que no apoyan la hipótesis de correlación y brindaron evidencia acerca de la ausencia de correlaciones claras entre los mecanismos cognitivos piagetianos y la comunicación intencional. Los dos dominios de instrumentalidad - el uso de instrumentos mecánicos y el uso de instrumentos semióticos - parecen desarrollarse con relativa independencia. En la actualidad, a la vez que se debilita la hipótesis de correlación entre inteligencia sensoriomotora y comunicación intencional, los gestos previos al habla empiezan a ser contemplados desde la perspectiva de los mecanismos cognitivos específicos que los niños ponen en juego en la interacción social, es decir, desde la perspectiva de las habilidades mentalistas implicadas en ellos (Goodhart y Baron Cohen, 1991; Gómez, Sarriá y Tamarit, 1993; Perucchini y Camaioni, 1993; Perucchini, 1997; Rivière, 1997; Gómez, y Núñez, 1999; etc.). La concepción de que a los primeros gestos subyace la noción del otro como agente de la acción es revisada y criticada, acentuándose la presencia de habilidades de cognición social en la comunicación intencional. Se sostiene que la producción de protodeclarativos requiere alguna conciencia de los otros no sólo como agentes sino como sujetos con quienes es posible compartir la experiencia (Phillips, Gómez, Baron-Cohen, Laa y Rivière, 1997; Rivière y Sotillo, 1998).

Entre los diversos gestos que producen los niños el gesto de señalar ha ocupado un lugar privilegiado en la literatura. Es posible que tal privilegio se deba a la pluralidad de funciones que se le han atribuido así como a la diversidad de hipótesis, acerca de su origen, que se han elaborado. Existen gestos que parecen ser usados con una única función: el gesto de mostrar se asocia específicamente con una función declarativa; el gesto de pedir ser alzado en brazos se vincula con la función imperativa; el señalar, en cambio, además de poder usarse tanto con una función imperativa como declarativa parece enlazarse con otra función específica que, si bien fue observada hace varias décadas, se ha tornado en los últimos años un tema polémico. Werner y Kaplan (1984) fueron los primeros en indicar una función puramente referencial del gesto de señalar; ellos sugirieron que el señalar es principalmente un mecanismo atencional perteneciente a los mecanismos que permiten percibir una imagen separando una figura de un fondo difuso. Unos años después E. Bates y colaboradoras $(1976,1979)$ comentaron haber observado que, antes de ligarse a situaciones comunicativas, el gesto de señalar es utilizado en situaciones no comunicativas, en esquemas de señalar para sí mismo. El estudio de la naturaleza de las habilidades sociales implicadas en el gesto de señalar para sí mismo ocupa actualmente un lugar destacado en la psicología del desarrollo. Algunas investigaciones reconocen su naturaleza no comunicativa (Camaioni, Perucchini, Murator y Milone, 1997); desde una postura opuesta, otro grupo de investigadores resalta la básica condición social de compartir que requiere el gesto de señalar para sí mismo. (Franco, Perucchini y Butterworth, 1992; Franco y Butterworth, 1996; Butterworth, 1998; Delgado, Gómez y Sarriá, 1999).

Si se puede hablar de una trilogía funcional del gesto de señalar — función imperativa, declarativa y no comunicativa - no menos puede decirse de las hipótesis explicativas de su origen. Éstas pueden agruparse en tres grupos: 1Aquéllas que apelan a ideas interaccionistas de corte vygotskiano y que consideran que el gesto surge mediante un proceso de ritualización de los intentos fallidos por parte del niño de coger un objeto en situaciones comunicativas en las cuales los adultos completan la acción iniciada por éste (Lock, 1980). 2- Aquéllas que sugieren que el gesto se construye mediante imitación (Kaye,1982; Car- 
penter, Nagell, y Tomasello, 1998). 3-Aquéllas que consideran que el gesto se origina a través de un cambio desde una función asocial a una social (Bates, 1976; Werner y Kaplan, 1984). Desde esta última perspectiva, el origen del gesto de señalar no se encuentra vinculado al acto de alcanzar un objeto - acto ligado al mundo pragmático de la acción — sino que se encuentra vinculado al acto de tocar un objeto - acto que se relaciona con el mundo de los objetos contemplados y con el reconocimiento de la existencia de un objeto situado a cierta distancia de uno mismo- ${ }^{1}$.

Las diversas hipótesis del origen del gesto y su pluralidad funcional se encuentran enlazados: la explicación de su origen basada en intentos fallidos de coger un objeto se encuentra en estrecha relación con la función imperativa con que puede usarse el gesto; por otro lado, tanto su función declarativa comunicativa, como la función "no comunicativa" referencial del señalar para sí mismo se vinculan más con las explicaciones que proponen que el gesto deviene del acto de tocar. Quienes sostienen que inicialmente el gesto de señalar sirve a propósitos declarativos más que imperativos y que sólo posteriormente puede adquirir también una función imperativa, suelen negar que el gesto provenga del acto de coger un objeto, más bien sugieren que ése acto genera otro gesto, el reaching, que es morfológica y funcionalmente diferente del gesto de señalar (Lock, Young, Service y Chandler, 1990; Franco y Butterworth, 1996; Butterworth, 1998). A su vez, quienes han observado y prestado atención al uso aparentemente no comunicativo del gesto en el que el niño parece dedicado a un acto epistémico o de contemplación del objeto - alejado de cualquier intención imperativa, aunque tal vez no de una delarativa - se encuentran inclinados a pensar que el gesto se deriva de la acción directa de tocar — no de coger - un objeto (Werner y Kaplan, 1984; Bates, 1976).

Íntimamente conectado con ambas cuestiones — su extraña polifuncionalidad y la prolífica discusión acerca de la naturaleza de la acción directa a partir de la cual emerge el gesto - se encuentra el hecho de que el estudio del gesto de señalar suele realizarse en relación con otros gestos relacionados o "vecinos" que se le asemejan morfológicamente y que se considera que pueden estar ontogenéticamente relacionados con él. El gesto de señalar se define como la extensión del brazo y dedo índice hacia un objetivo que — aunque no siempre se señalecoincide con la dirección de la mirada del niño. Desde los trabajos de Shaffer (1984) se reconoce que usualmente aparece en el desarrollo entre los doce y los catorce meses. (Aunque existen registros de una producción del gesto, si bien menos frecuente, en niños de diez meses de edad (Franco y Butterworth, 1996)).

Una morfología similar o vecina al gesto de señalar es la que se ha identificado como reaching o "gesto de extenderse hacia". En ella el brazo se encuentra extendido con la palma de la mano hacia abajo y abierta. Algunos investigadores, sobre la base de observaciones que señalaban un incremento del gesto de señalar y una disminución del reaching entre los nueve y los dieciocho meses, sugirieron que el gesto de señalar paulatinamente reemplaza al reaching en el desarrollo (Murphy y Messser, 1977; Murphy, 1978; Leung y Rheingold, 1981). Sin embargo, Franco y Butterworh (1996), considerando que estos estudios dejaban en la oscuridad las situaciones contextuales en la que se producían los gestos y el tipo de estímulo utilizado, diseñaron dos condiciones experimentales 1-: una "condición declarativo-referencial" en la que se promovía la intención de compartir con la madre el interés hacia la situación estimular, sin que necesariamente ésta indujera en el niño el deseo de agarrar el objeto y 2-: una "condición imperativo-instrumental” diseñada para elicitar la intención de manipular un juguete y pedir ayuda al adulto para poder alcanzarlo. Observaron que el gesto de señalar 
fue usado principalmente en la condición referencial y el reaching en la condición instrumental. Interpretaron sus resultados como una evidencia en contra de que el origen del gesto de señalar se encuentre en la sustitución del reaching y a favor de que las funciones de los dos gestos se originan desde contextos diferentes de comunicación: declarativo-referencial para el gesto de señalar, e imperativo-instrumental para el reaching.

El gesto de señalar se encuentra relacionado con otro gesto: el "gesto indicativo" en el cual el brazo se encuentra extendido pero se pierde la extensión del dedo índice. La frecuencia de este gesto, si bien baja, parece permanecer estable en el desarrollo lo que ha llevado descartar la hipótesis de que éste sea paulatinamente reemplazado por el gesto de señalar y ha favorecido la idea de que éste es un gesto particular — no un precursor del gesto de señalar- con una función propia. (Lock, Young, Service y Chandler, 1990; Franco y Butterworth, 1996). Sin embargo, es poco lo que sabemos acerca de él: Lock, Young, Service y Chandler (1990) sugieren que el gesto indicativo tiene una función diferente del gesto de señalar, pero no indican cuál es; Franco y Butterworth (1996) sostienen que el gesto indicativo puede considerarse funcionalmente equivalente al gesto de señalar ya que ambos tienden a producirse en contextos declarativos referenciales, a diferencia del reaching que es más frecuente en contextos imperativos instrumentales. La escasa información acerca del gesto se une a que existen opiniones diversas en cuanto a las variedades morfológicas que agrupa. Franco y Butterworth (1996) incluyen como formando parte de los gestos indicativos una clase de señalar que se realiza muy rápido, con el dedo índice extendido, pero sin que llegue a extenderse el brazo y sin que coincidan la dirección del brazo y el dedo y la dirección de la mirada. Este gesto rápido, denominado point slips out, aparece entre los cinco y los ocho meses y luego decrece hasta casi desaparecer a los trece meses (Lock, Young, Service y Chandler, 1990). Nos parece más adecuado, tal y como hacen Lock et al., no incluir en la categoría de gesto indicativo a este último gesto cuya morfología y evolución es tan diferente.

Cuando se estudia el desarrollo del gesto de señalar suele reconocerse que los niños, además del gesto de señalar prototípico, realizan producciones sígnicas semejantes a él morfológicamente. Sin embargo, se han utilizado criterios diferentes a la hora de estudiarlos. Estos criterios reflejan si las diversas morfologías son consideradas gestos particulares - lo cual lleva a indagar acerca de sus funciones específicas-, o si éstas son vistas como variedades morfológicas de un único gesto. Algunos investigadores tratan al gesto de señalar de un modo global, incluyendo todas las variedades morfológicas en una misma categoría y restringiendo su definición a "la extensión del brazo hacia un objeto con o sin la extensión del dedo índice” (Carpenter, Nagell y Tomasello, 1998). Otros abogan por que se diferencie entre ellos y que se reserve la denominación de gesto de señalar a la extensión simultánea del brazo y el dedo índice (Lock, Young, Service y Chandler, 1990; Franco y Butterworth, 1996; Butterworth, 1998; etc).

En síntesis, hemos hablado de la morfología, el origen y la función del gesto de señalar, y al hacerlo nos referirnos a los dos gestos que se consideran (o que se discuten si están) ontogenéticamente relacionados con él: el reaching y el gesto indicativo. Hemos señalado, también, los diferentes criterios utilizados en su estudio. Nos queda una última cuestión por tratar y es pensar acerca del modo como se satisfacen las distintas funciones del gesto. Podemos decir que la intención comunicativa imperativa del señalar se satisface en la medida en que se producen los cambios en el mundo que quien señala pretende; la intención comunicativa de las pautas protodeclarativas sólo se satisface en la mente 
de los otros y en la medida en que se producen en ella procesos que implican compartir intersubjetivamente intereses. Es decir, los protoimperativos buscan, en último término, cambiar el mundo físico; los protodeclarativos pretenden cambiar el mundo mental del otro (Rivière,1997). Una cuestión semióticamente interesante es que cuando se pretende describir conductas comunicativas resulta casi inevitable recurrir a la descripción de la mirada, específicamente a los fenómenos de contacto ocular y atención conjunta (Scaife y Bruner, 1975; Trevarthen y Hubley, 1978; Morisette, Ricard y Gouin Décarie, 1995; Carpenter, Nagell y Tomasello, 1998; Butterworth, 1998; etc). Estos fenómenos son uno de los elementos más primitivos con los que los seres humanos nos relacionamos con nuestros congéneres y con los objetos y sucesos del mundo. Durante el primer y segundo año de vida, se presentan cambios en los patrones de la mirada del niño que sugieren modificaciones en su significado psicológico (D’odorico y Levorato, 1990). La dinámica de la mirada del niño y del adulto cobra diversos matices según la función del gesto; en las pautas protoimperativas el niño busca la mirada del otro para asegurarse su atención y pedir lo que desea; en las pautas protodeclarativas, en cambio, es en el acto mismo de mirarse el uno al otro y al objeto (y en las declaraciones que suelen acompañarlo) que se satisface la función. ¿Qué sucede en el señalar no comunicativo en el que ocurre un fenómeno semiótico particular y es que el emisor y el interpretante del gesto confluyen en un mismo sujeto?, ¿cómo se satisface el gesto si éste tiene una función básicamente referencial?. Y, ¿qué ocurre con los gestos relacionados con el gesto de señalar?, ¿existen patrones de miradas relacionados con cada uno de ellos? Los datos de Franco y Butterworth (1996) indican patrones de mirada diferentes para el gesto de señalar y el reaching, aunque nada dicen acerca de los gestos indicativos. Una última cuestión, los adultos tenemos un manejo escandalosamen te sofisticado de la mirada; ¿qué ocurre en edades tempranas?, ¿qué sucede cuando el contexto interpersonal en el que se encuentra el niño varía?, ¿adecuan el uso de su mirada a la situación comunicativa en la que se encuentran?, ¿la variedad de contextos interpersonales afecta otros aspectos de la producción del gesto de señalar? En este trabajo intentaremos responder a algunas de estas preguntas centrándonos en los gestos con función declarativa.

\section{MÉTODO}

\section{Objetivos}

El trabajo se orienta a estudiar el desarrollo de la función declarativa del gesto de señalar y, más específicamente, a observar si la producción del gesto es sensible al contexto interpersonal en el que se produce. Nuestro interés es observar tanto el comportamiento del gesto de señalar considerado de modo global como los comportamientos específicos del gesto de señalar prototípico y de sus gestos vecinos.

\section{Participantes}

Se trabajó con una muestra de 27 niños divididos en tres grupos de edad de 9 niños cada uno. Los grupos de edad fueron de 10 meses (4 varones y 5 niñas), 13 meses ( 3 varones y 6 niñas) y 16 meses ( 4 varones y 5 niñas). 


\section{1}

\section{Materiales}

\section{A-Condiciones experimentales}

Se diseñó una condición experimental general, con el fin de elicitar la producción del gesto de señalar y orientada a favorecer su función declarativo referencial, y tres situaciones particulares en las que se variaron los grados de contacto interpersonal. La situación experimental general fue semejante a la condición experimental declarativo-referencial de Franco y Butterworth (1996). En ella se buscó despertar la intención del niño de compartir su interés por la situación estimular con el adulto. Se colocó sobre una mesa, en frente del niño y a una distancia de dos metros del niño, un teatro con luces intermitentes del que entraba y salía a intervalos regulares un objeto.

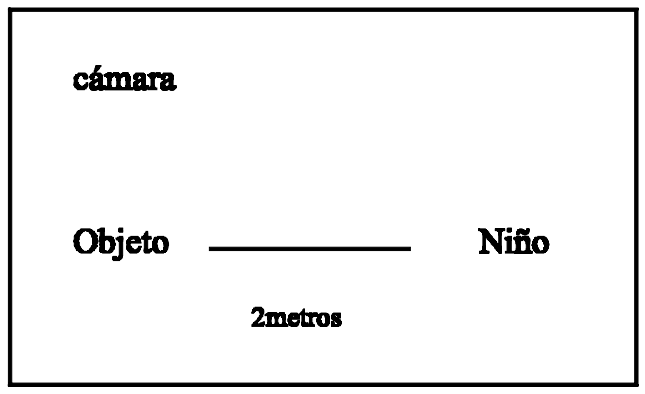

Se diseñaron tres condiciones experimentales en las que varió el grado de contacto interpersonal entre el niño y el adulto.

1. Situación de alto contacto interpersonal: el niño se encuentra en brazos del adulto.

2. Situación de nivel medio de contacto interpersonal: el niño se encuentra sentado en un silla alta y el adulto se encuentra a su lado en un ángulo de $90^{\circ}$.

3. Situación de bajo nivel de contacto interpersonal: el niño se encuentra sentado en un silla alta y el adulto se encuentra detrás, a dos metros de distancia.

\section{1. niño en bramos del adulto}
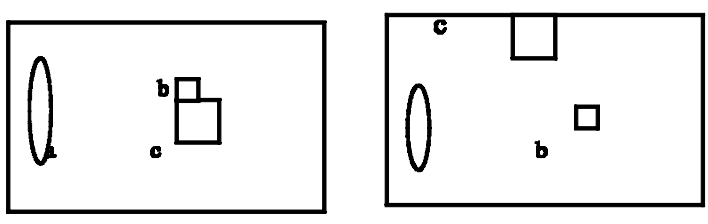

\section{3. niiño con el aculto detrás}

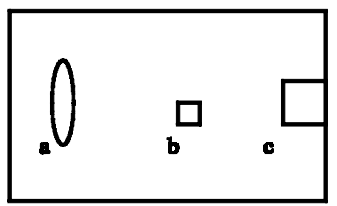

a: teatro; b: niño; c: aculto

En las tres condiciones experimentales la situación general de estimulación fue la misma.

Dado que cada niño pasó por las tres situaciones experimentales, se construyeron tres objetos equivalentes. Se mantuvo constante en las tres situaciones el teatro con luces intermitentes del cual entraba y salía, a intervalos regulares, un objeto. En cada situación se varió únicamente el objeto. Los tres objetos tuvieron forma, color, sonido y movimiento diferente. En las tres situaciones la secuencia 
total de estimulación fue de tres minutos en los que se presentaba la siguiente secuencia:

1. de 00:00 sa 00:30 s

2. de 00:30s a 00:50 s.

3. de 00:50s a $1 \mathrm{~m}: 30 \mathrm{~s}$.

4. de $1 \mathrm{~m}: 30 \mathrm{~s}$ a $1 \mathrm{~m}: 50 \mathrm{~s}$

5. de $1 \mathrm{~m}: 50 \mathrm{~s}$ a $2 \mathrm{~m}: 10 \mathrm{~s}$

6. de $2 \mathrm{~m}: 10 \mathrm{~s}$ a $2 \mathrm{~m}: 30 \mathrm{~s}$

7. de $2 \mathrm{~m}: 30 \mathrm{~s}$ a $3 \mathrm{~m}: 00 \mathrm{~s}$

8. $3: 00 \mathrm{~m}$
Teatro vacío con luces intermitentes.

Sonido del objeto que en ese momento no se ve.

Entra el objeto en el escenario con sonido y movimiento.

Sale el objeto del escenario y continúa el sonido del objeto.

Silencio.

Sonido del objeto.

Entra el objeto con sonido y movimiento.

Sale el objeto.

El orden de presentación de las condiciones experimentales y de los objetos se controló mediante la técnica de cuadrado grecolatino.

\section{B. Códigos de observación}

En función de la configuración adoptada por brazo, mano y dedos del niño se identificaron tres gestos:

1. Gesto de señalar prototípico: extensión del brazo y dedo índice hacia un objetivo.

2. Reaching: extensión del brazo con la palma de la mano hacia abajo y abierta hacia un objetivo.

3. Gesto indicativo: extensión del brazo con los dedos apenas flexionados y el dedo índice sobresaliendo levemente hacia un objetivo.

Las primeras dos categorías son semejantes a las de Franco y Butterworth (1996); la última difiere de la de ellos en dos aspectos: 1 - no incluimos como gesto indicativo al point slip out (clase de señalar que se realiza muy rápido, con el dedo índice extendido pero sin que llegue a extenderse el brazo ni que haya coincidencia con la dirección del gesto y la dirección de la mirada) por los motivos expuestos en la introducción. 2- Franco y Butterworth (1996) definen esta categoría, de un modo bastante general, como la extensión del brazo mientras la mano asume diversas posturas (la mano enteramente extendida o con los dedos levemente flexionados) (Lock et al., 1990) también dan una definición general gesto: extensión del brazo con pérdida de extensión del dedo índice. En un estudio longitudinal de caso único previo (Español, 1999), observamos que, en los casos en que se configuraba el gesto con la extensión del brazo pero sin la extensión del dedo índice, la mano solía adoptar una morfología particular: los dedos se encontraban sin tensión, apenas flexionados, y el dedo índice, pese a no estar en extensión, se encontraba un poco por encima del nivel de los otros dedos. Dado que se detectó la presencia de una forma altamente frecuente, clara, y diferenciable de otras, se decidió incluir sólo ésta bajo el rótulo "gesto indicativo".

Como el gesto de señalar, cuando tiene una función sígnica, no se constituye únicamente con la morfología de brazo y mano sino que la dirección de la mirada se encuentra incluida en su configuración, se analizó la producción del gesto de señalar tomando en cuenta la dirección de la mirada del niño. Se consideró la dirección de la mirada del niño en el momento de ejecución del gesto y durante los tres segundos anteriores o posteriores a su realización. Se identificaron tres tipos de configuraciones.

1. Tipo "solo": el gesto es acompañado sólo por la dirección de la mirada del niño hacia el mismo lugar hacia el que dirige su gesto. 
2. Tipo "secuencial”: el niño, además de realizar el gesto mirando hacia el mismo lugar al que dirige su gesto, establece contacto ocular con el adulto inmediatamente antes o inmediatamente después de realizar el gesto.

3. Tipo "alternado": el niño además de realizar el gesto mirando hacia el mismo lugar al que dirige su gesto, establece contacto ocular con el adulto antes y después de realizar el gesto, o durante su ejecución.

\section{Diseño y procedimiento}

La investigación se realizó desde una doble perspectiva. En lugar de elegir alguna de las dos estrategias de investigación del gesto de señalar reseñadas en la introducción, se decidió realizar su estudio desde dos aproximaciones al análisis de los datos. En la primera se estudió la producción del gesto de señalar como categoría global, sin distinguir entre las diversas configuraciones morfológicas. Se categorizó como gesto de señalar a la extensión del brazo hacia el estímulo, con o sin extensión del dedo índice. En la segunda aproximación se estudió el comportamiento del gesto de señalar, diferenciando entre éste y sus gestos vecinos.

En la primera aproximación la investigación responde a un diseño factorial complejo de $3 \times 3 \times 3$ en el que se estudia la producción del gesto de señalar en función de la edad, de las tres situaciones y de los tres modos de coordinación del gesto con la mirada. La variable independiente "edad" contempla tres niveles: 10 meses, (edad media 0;10.03; desviación típica 0;0,5); 13 meses (edad media $1 ; 1.02$; desviación típica $0 ; 0,13$ ), y 16 meses (edad media 1;4.05; desviación típica $0 ; 0,8)$. La variable independiente intrasujeto "situación” contempla las tres situaciones experimentales diseñadas: situación "en brazos", de alto contacto interpersonal; situación "al lado", de contacto interpersonal medio y situación "detrás" de bajo contacto interpersonal. La variable independiente de medidas repetidas "tipo" es el resultado de aplicar el código de observación en relación con los tres tipos observados: "solo", "secuencial" y "alternado". La variable dependiente fue la frecuencia del gesto de señalar. Se registraron únicamente los gestos dirigidos al objeto estímulo.

En la segunda aproximación se realizó un análisis factorial complejo de 3×3×3 con el objetivo de estudiar la producción del gesto de señalar y de sus dos gestos vecinos, en función de las tres situaciones y en función de los tres modos de coordinación del gesto con la mirada. Se incluyó, además de las variables independientes presentes en la primer aproximación ("situación” y "tipo"), la variable independiente "morfología". La variable independiente de medidas repetidas "morfología” es el resultado de aplicar el código de observación. Los niveles de variable corresponden a los tres gestos identificados (Gesto de señalar, reaching y gesto indicativo). Al igual que en la primer aproximación la variable dependiente fue la frecuencia de los gestos registrándose únicamente los gestos dirigidos al objeto estímulo. La variable "edad" quedó eliminada en función de los resultados obtenidos en la primera aproximación (ver punto siguiente)

Los niños fueron visitados en su guardería y en todos los casos el adulto que estuvo con ellos fue su cuidadora. (Salvo en dos casos de niños de 10 meses que fueron visitados en sus hogares y estuvieron con sus madres). A las cuidadoras (y a las madres) se les dijo que respondieran al bebé normalmente, salvo en la "situación detrás" en la que se les pidió que hicieran como "que no estaban" a no ser que el niño reclamara su presencia. Los niños fueron observados de forma individual y con un intervalo de por lo menos diez minutos entre cada situación. Las sesiones experimentales se grabaron en cintas de vídeo con una cámara fija. El registro de las observaciones se realizó sobre la base de las cintas de vídeo. 


\section{RESULTADOS}

\section{Primera aproximación al análisis de los datos}

Se comparó la producción del gesto de señalar, considerado de forma global, en los tres grupos de edad, en función de las tres situaciones y de los tres tipos de coordinación de gesto y mirada. Los datos concernientes a las frecuencias de los gestos y tipo de gestos en las tres condiciones se obtuvieron mediante medidas repetidas de análisis de variancia.

Siguiendo las recomendaciones de León y Montero (1997) para el análisis de resultados en diseños complejos, se empezó estudiando la interacción de orden superior. Los datos muestran una interacción triple "edad-tipo-situación" $\mathrm{F}(8,96)=2,156, \mathrm{p}<.08)^{2}$. Sin embargo, analizados los tres grupos de edad de modo separado se observa que en el grupo de edad de 10 meses la ocurrencia del gesto de señalar es escasa y no se detectan efectos de interacción simple ni efectos principales estadísticamente significativos. Con el fin de averiguar si la interacción triple muestra sólo un efecto suelo a los 10 meses, se realizó un nuevo Anova en diseño $2 \times 3 \times 3$ incluyendo "edad" (13 y 16 meses) como variable intersujeto, "situación" (en brazos, al lado, detrás, ) y "tipo" (solo, secuencial y alternado) como variables intrasujetos. En este diseño la interacción triple "situación-edad-tipo" no es estadísticamente significativa $(\mathrm{F}(4,64)=0,770, \mathrm{p}>.10)$. Dados estos resultados se realizó un nuevo Anova sin incluir la variable "edad". En este análisis aparece una interacción significativa "situación-tipo" $(F(4,68)=6,254$, $\mathrm{p}<.01$ ). La figura 1 muestra la forma de la interacción (ver Figura 1).

Dado que en ambas gráficas la forma de la interacción es no ordinal, se procedió a estudiar los efectos simples de las variables "situación” y "tipo". Empezaremos con el análisis de los efectos de la variable "tipo". El primero de ellos, el efecto de la variable "tipo" sobre el número de gestos condicionado a la situación «en brazos» muestra que existe una diferencia en la cantidad de gestos de señalar $(\mathrm{F}(2,34)=14,43, \mathrm{p}<.001)$. La comparación entre pares muestra diferencia entre tipo "solo" y tipo "secuencial” $\left(\mathbf{M}_{\text {solo }}: 2,61 v \text { s. } \mathbf{M}_{\text {secuencial }}: 0,17, \mathrm{p}<.001\right)^{3}$ y entre el tipo "solo" y tipo "alternado" ( $\mathbf{M}_{\text {solo }}: 2,61 v$ s. $\left.\mathbf{M}_{\text {alternado }}: 0,22, \mathrm{p}<.01\right)$. Los dos efectos restantes, es decir, los efectos la variable "tipo" sobre el número de gestos condicionado a la situación «al lado" y "detrás” no resultan significativos. Continuamos con el análisis de los efectos de la variable "situación" El primero de ellos, el efecto de la variable "situación" sobre el número de gestos condicionado al tipo "solo" muestra que existe una diferencia en la cantidad de gestos de señalar $(\mathrm{F}(2,34)=11,22, \mathrm{p}<.001)$. La comparación entre pares muestra diferencia entre la situación «en brazos» y «al lado» $\left(\mathrm{M}_{\text {en brazos }}: 2,61\right.$ vs. $\left.\mathrm{M}_{\text {al lado }}: 1,33, \mathrm{p}<.01\right)$; entre la situación «en brazos» $\mathrm{y}$ «detrás» $\left(\mathrm{M}_{\text {en brazos }}: 2,61\right.$ vs. $\left.\mathrm{M}_{\text {detrás }}: 0,17, \mathrm{p}<.01\right)$ y entre la situación «al lado» $\mathrm{y}$ «detrás» $\left(\mathrm{M}_{\text {al lado }}: 1,33\right.$ vs. $\mathrm{M}_{\text {derrás: }}$ 0,17, $\left.\mathrm{p}<.01\right)$. El efecto simple de la "situación" condicionado al tipo "secuencial" muestra que existe una diferencia en la cantidad de gestos de señalar $(\mathrm{F}(2,34)=5,651, \mathrm{p}<.05)$. La comparación entre pares muestra diferencia entre la situación «en brazos» $\mathrm{y}$ «al lado» $\left(\mathrm{M}_{\text {en brazos }}: 0,17\right.$ vs. $\left.\mathrm{M}_{\text {al lado }}: 0,94, \mathrm{p}<.03\right)$; y entre la situación «al lado» $\mathrm{y}$ «detrás» $\left(\mathrm{M}_{\text {al lado }}: 0,94\right.$ vs. $\left.\mathrm{M}_{\text {detrás: }}: 0, \mathrm{p}<.02\right)$. El efecto simple de la "situación" condicionado al tipo "alternado" muestra que existe una diferencia en la cantidad de gestos de señalar en función de la situación $(\mathrm{F}(2,34)=3,966, \mathrm{p}<.06)$. La comparación entre pares muestra una diferencia entre la situación «al lado» $\mathrm{y}$ «detrás» $\left(\mathrm{M}_{\text {al lado }}\right.$ : 1,22 vs. $\left.\mathrm{M}_{\text {detrás }}: 0, \mathrm{p}<.04\right)$.

El análisis de los datos sugiere que:

A los 10 meses la ocurrencia del gesto de señalar es escasa y no se presentan interacciones ni efectos estadísticamente significativos. Entre los 13 y los 16 


\section{5}

FIGURA 1

Efecto de interacción simple. Arriba: Interacción situación-tipo. Abajo: Interacción tipo-situación
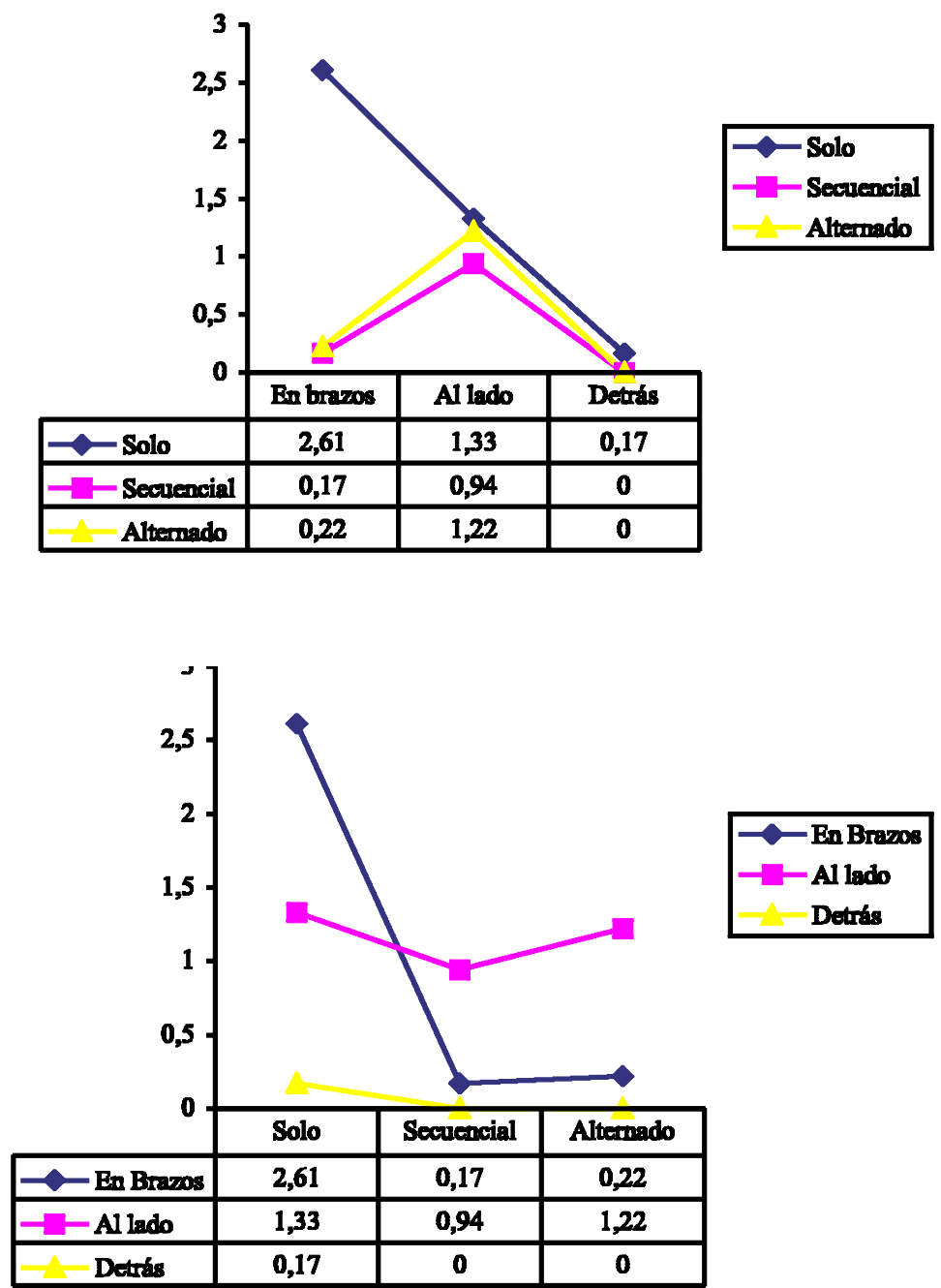

meses no hay variaciones estadísticamente significativas en la ocurrencia del gesto de señalar ni en función del tipo ni en función de la situación.

En el período comprendido entre los 13 y 16 meses los niños se muestran sensibles al contexto comunicativo siendo las situaciones con alto y medio contacto interpersonal (en brazos y al lado) las que más elicitan la producción del gesto de señalar. Más específicamente: 1- La situación "en brazos" elicita principalmente un señalar sin búsqueda de contacto ocular. 2- La situación "al lado" elicita tres tipos de gestos sin que llegue a haber diferencias significativas entre ellos. Es decir, en esta situación, los niños son capaces de realizar el gesto alternando el gesto con la mirada; realizan también el gesto acompañado de un chequeo visual inmediatamente antes o después de la realización del gesto y realizan también el gesto sin que lo acompañe ningún tipo de chequeo visual. 3- En la situación 


\section{6}

"detrás" no se presentan diferencias significativas entre los tres tipos de gestos, pero esta ausencia de significatividad se debe a que la situación sólo elicita el gesto tipo "solo" con una frecuencia que no llega a ser estadísticamente significativa. Pese a ello, y como veremos más adelante, la producción escasa de gestos de este tipo en la situación detrás es relevante desde un punto de vista teórico.

Dijimos que entre los 13 y 16 meses no hay una diferencia estadísticamente significativa en la producción del gesto de señalar; sin embargo, aparece una inversión, cualitativamente relevante, en la frecuencia de producción del tipo "secuencial" y "alternado", en la situación "al lado". A los 13 predomina un uso secuencial de mirada y gesto mientras que a los 16 se acentúa la alternancia entre el gesto y la mirada. Las figuras 2 y 3 muestran esta inversión.

FIGURA 2

Gesto de señalar a los 13 meses

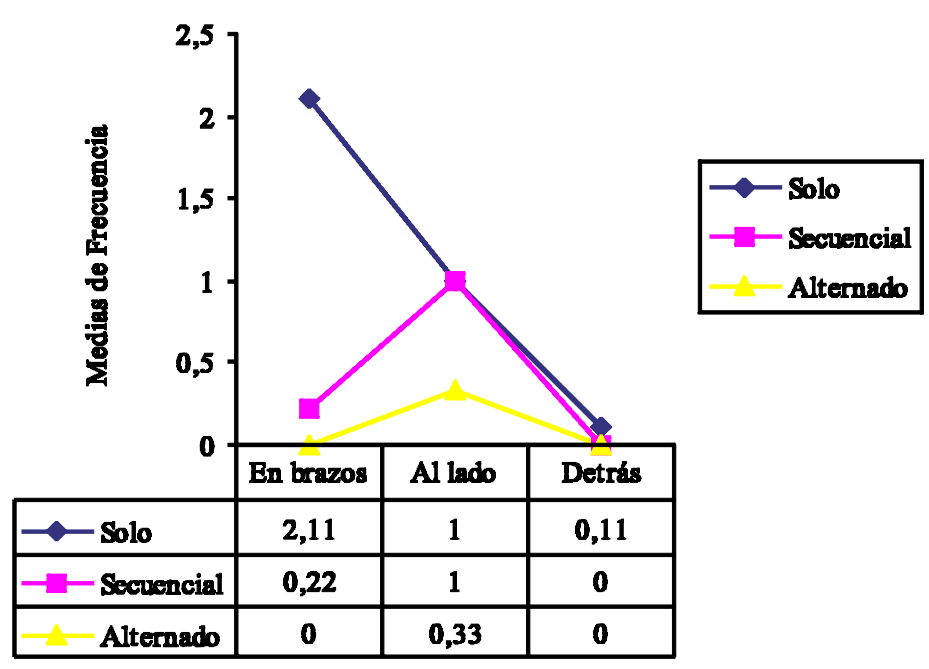

FigURA 3

Gesto de señalar a los 16 meses
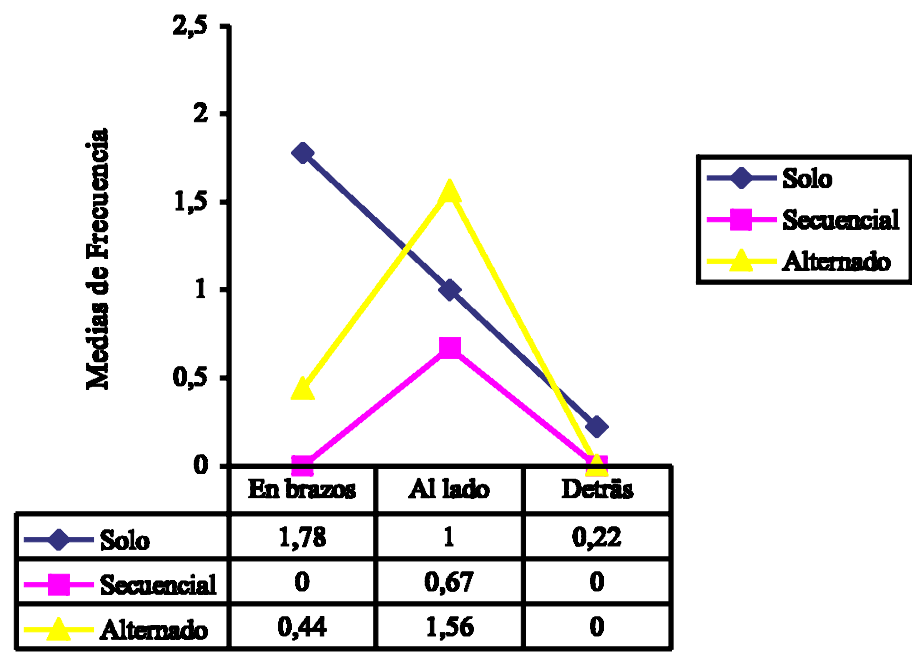
Segunda aproximación al análisis de los datos

En esta segunda aproximación el estudio responde a un diseño factorial complejo de $3 \times 3 \times 3$ en el que se estudia la producción del gesto de señalar, del reaching y del gesto indicativo, en función de las tres situaciones y en función de tres modos de coordinación del gesto con la mirada Dado los resultados obtenidos en la primera aproximación no se incluyó la variable «edad» y se aunó en uno solo a los grupos de 13 y 16 meses.

La frecuencia de los de las tres gestos fue: 90 gestos de señalar (79\%); 15 gestos indicativos $(13 \%)$ y 10 reaching $(8 \%)$. Al igual que en la primera aproximación, los datos concernientes a las frecuencias de los gestos y tipo de gestos en las tres condiciones se obtuvieron mediante medidas repetidas de análisis de variancia. El análisis en diseño de 3×3×3 incluye la situación ("en brazos", "al lado" y "detrás"); el tipo ("solo", "secuencial” y "alternado”) y la morfología ("gesto de señalar prototípico", "reaching" y "gesto indicativo") como variables intrasujetos.

El estudio de la interacción de orden superior muestra una interacción estadísticamente significativa "tipo-situación-morfología" $(\mathrm{F}(8,136)=2,446$, p < .09). Aunque el análisis de la interacción triple permite diferentes variantes, en función de los objetivos de este trabajo hemos considerados el análisis de la interacción "situación-tipo" en cada una de las tres morfologías.

Gesto de señalar prototípico: Los datos indican una interacción significativa “tipo-situación” en el gesto de señalar $(\mathrm{F}(4,68)=3,885, \mathrm{p}<.05)$. La figura 4 muestra la forma de la interacción.

\section{FIGURA 4}

Efecto de la interacción simple para el caso del gesto de señales prototípico

Interacción situación-tipo

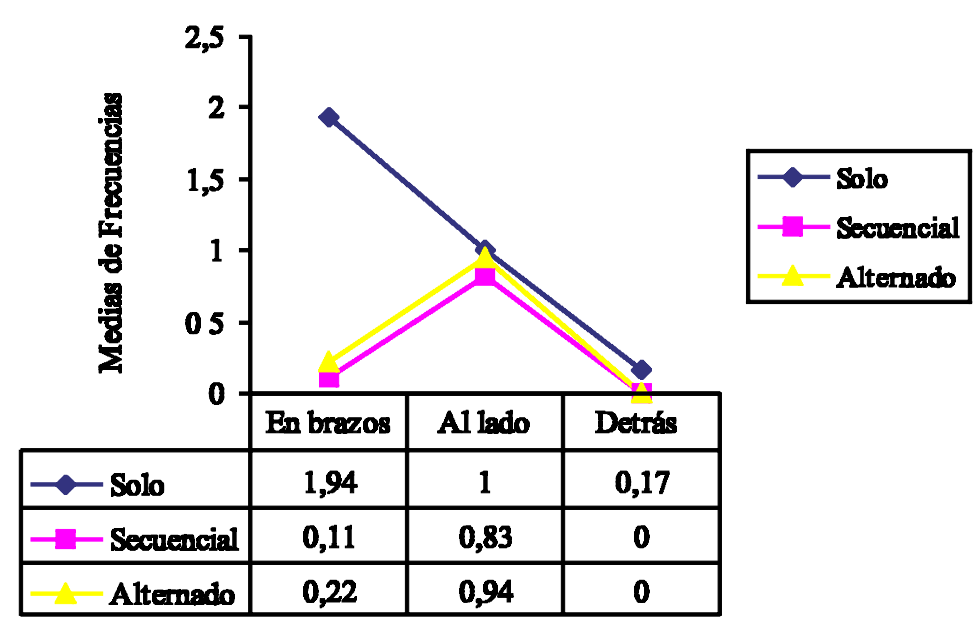



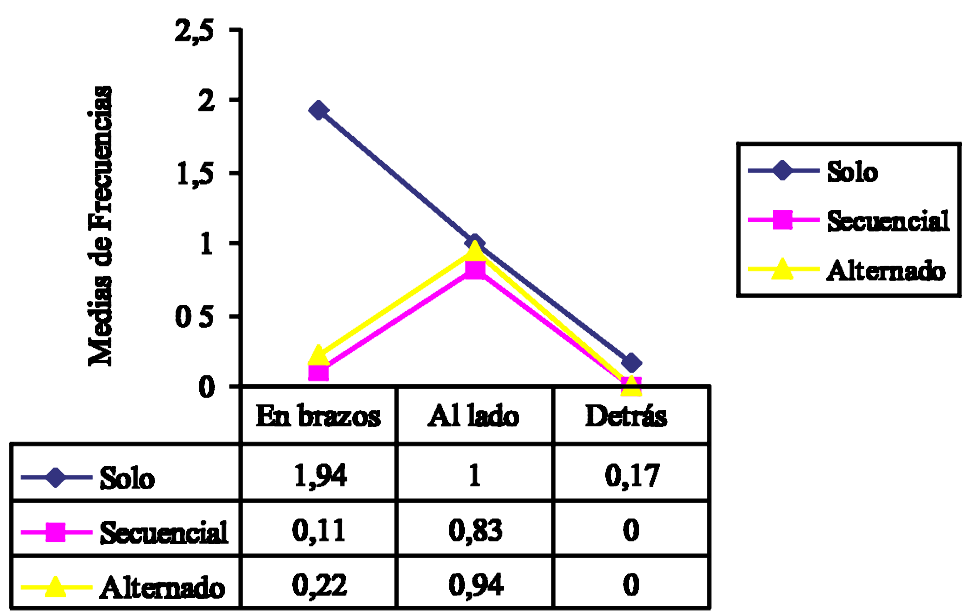

Dado que en ambas gráficas la forma de la interacción es no ordinal, se procedió a estudiar los efectos simples de las variables "situación” y "tipo". Empezaremos con el análisis de los efectos de la variable "tipo". El primero de ellos, el efecto de la variable "tipo" sobre el número de gestos condicionado a la situación «en brazos» muestra que existe una diferencia en la cantidad de gestos de señalar prototípico $(\mathrm{F}(2,34)=11,555, \mathrm{p}<.01)$.La comparación entre pares muestra diferencia entre tipo "solo" y tipo "secuencial” $\left(\mathbf{M}_{\text {solo }}: 1,94 v\right.$ s. $\left.\mathbf{M}_{\text {secuencial }}: 0,11, \mathrm{p}<.01\right)$ y entre el tipo "solo" y tipo "alternado" ( $\mathbf{M}_{\text {solo }}: 1,94$ vs. $\left.\mathbf{M}_{\text {alternado }}: 0,22, \mathrm{p}<.01\right)$. Los dos efectos restantes, es decir, los efectos la variable "tipo" sobre el número de gestos condicionado a la situación «al lado" y "detrás” no resultan significativos. Continuamos con el análisis de los efectos de la variable "situación”. El primero de ellos, el efecto de la variable "situación" sobre el número de gestos condicionado al tipo "solo" muestra que existe una diferencia en la cantidad de gestos de señalar prototípico $(\mathrm{F}(2,34)=8,321, \mathrm{p}<.001)$. La comparación entre pares muestra diferencia entre la situación «en brazos»y «al lado» $\left(\mathbf{M}_{\text {en brazos }}: 1,94\right.$ vs. $\mathbf{M}_{\text {al lado }}$ : $1, \mathrm{p}<.01)$; entre la situación «en brazos» $\mathrm{y}$ «detrás» $\left(\mathrm{M}_{\mathrm{en} \text { brazos }}: 1,94\right.$ vs Mdetrás: $0,17, \mathrm{p}<.01)$. Los dos efectos simples restantes no resultan significativos, es decir la variable situación no parece influir al hablar de gestos secuenciales y alternados.

Gesto Indicativo: Se presenta una interacción significativa «tipo-situación» $(\mathrm{F}(4,68)=2,7771, \mathrm{p}<.09)$. La figura 5 muestra la forma de la interacción (ver Figura 5).

Dado que en ambas gráficas la forma de la interacción es no ordinal, se procedió a estudiar los efectos simples de las variables "situación” y "tipo". Empezaremos con el análisis de los efectos de la variable "tipo". El primero de ellos, el efecto de la variable "tipo" sobre el número de gestos condicionado a la situación «en brazos» muestra que existe una diferencia en la cantidad de gestos indicativos $(\mathrm{F}(2,34)=4,613, \mathrm{p}<.05)$. La comparación entre pares muestra diferencia entre tipo "solo" y tipo "secuencial” $\left(\mathrm{M}_{\text {solo }}: 0,50\right.$ vs. $\left.\mathbf{M}_{\text {secuencial }}: 0 \mathrm{p}<.03\right)$ y entre el tipo "solo" y tipo "alternado" ( $\mathbf{M}_{\text {solo }}: 0,50$ vs. $\mathbf{M}_{\text {alternado }}$ : $\left.0, \mathrm{p}<.03\right)$. El efecto simple del 


\section{9}

FigURA 5

Efecto de interacción simple para el caso del gesto indicativo. Arriba: Interacción situación-tipo. Abajo: Interacción tipo-situación
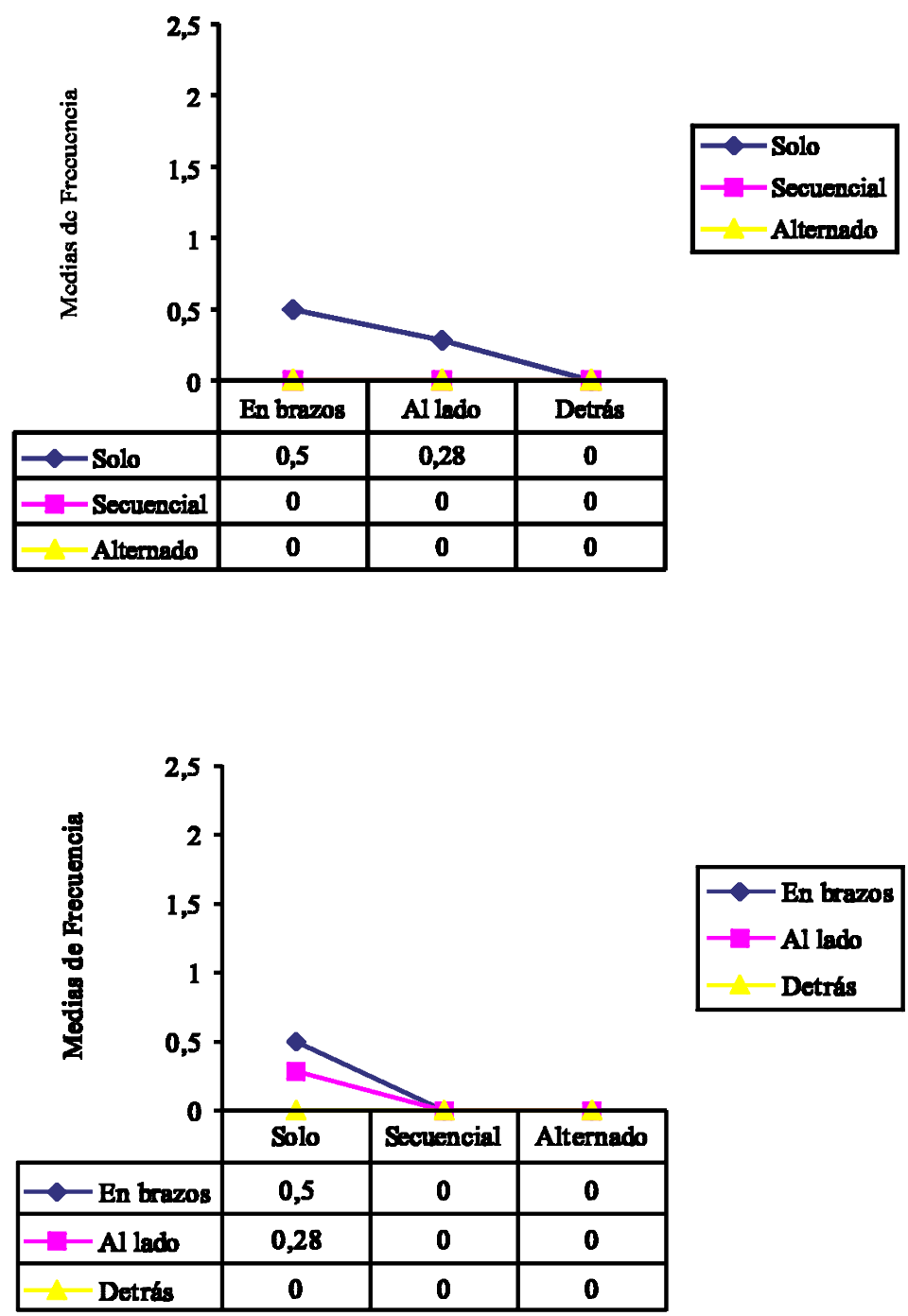

"tipo" condicionado a la situación «al lado» muestra que existe una diferencia en la cantidad de gestos indicativos $(\mathrm{F}(2,34)=4,208, \mathrm{p}<.06)$. La comparación entre pares muestra diferencia entre tipo "solo" y tipo "secuencial" ( $\mathbf{M}_{\text {solo }}: 0,28 v s . \mathbf{M}_{\text {secuen- }}$ cial: $0, \mathrm{p}<.05)$, y entre el tipo "solo" y tipo "alternado" $\left(\mathrm{M}_{\text {solo }}: 0,28 v\right.$ s. $\mathrm{M}_{\text {alternado }}$ : 0 , $\mathrm{p}<.05)$. Continuamos con el análisis de los efectos de la variable "situación". Únicamente se manifiesta el efecto de la variable "situación" sobre el número de gestos indicativos condicionado al tipo "solo". $(\mathrm{F}(2,34)=3,096, \mathrm{p}<.08)$. La comparación entre pares muestra diferencia entre la situación «en brazos» $\mathrm{y}$ «detrás» $\left(\mathrm{M}_{\text {en brazos }}: 0,50\right.$ vs. $\left.\mathrm{M}_{\text {detrás: }}: 0, \mathrm{p}<.03\right)$. 
En cuanto a la tercera morfología, el reaching, la interacción «tipo-situación» no es significativa y no se manifiesta tampoco ningún efecto principal.

El análisis de los datos sugiere que:

La mayoría de las configuraciones gestuales producidas por los niños, en las condiciones diseñadas por nosotros, responde a la categoría gesto de señalar prototípico. Los datos obtenidos al observar el funcionamiento de esta categoría particular son similares a los que se obtuvieron en la primera aproximación para el gesto de señalar considerado de modo global. En cuanto al reaching, no se presentan interacciones ni efectos estadísticamente significativos, probablemente debido a su escasa producción. La producción del gesto indicativo también es escasa, aunque levemente mayor que la del reaching. Pese a ello, el gesto muestra un comportamiento particular: se presenta únicamente bajo el tipo "sólo" de relación gesto-mirada y en contextos de alto y medio contacto interpersonal.

Interesados por estas particularidades del gesto indicativo, que se encuentran claramente asociadas con el comportamiento de lo que suele denominarse "señalar para sí mismo", se decidió investigar: 1- si había alguna diferencia en el comportamiento del gesto indicativo entre los 13 y los 16 meses. 2- si había alguna diferencia en el comportamiento de aquello que pueda ser considerado "gesto autodirigido" entre los 13 y los 16 meses. Esta clase engloba el gesto indicativo y el gesto de señalar prototípico únicamente de tipo "solo" en las situaciones "detrás" y "al lado" (ver la discusión para su justificación). Se realizó una prueba t para comparar las medias de frecuencia del gesto indicativo a los 13 y 16 meses. La comparación entre pares no muestra una diferencia significativa $\left(\mathrm{M}_{13}: 0,56 v \mathrm{~s} . \mathrm{M}_{16}: 1, \mathrm{p}>.10\right)$. Se realizó otra prueba t para comparar las medias de frecuencia de «gestos autodirigidos» a los 13 y 16 meses. La comparación entre pares no muestra una diferencia significativa $\left(\mathrm{M}_{13}: 1,44\right.$ vs. $\left.\mathrm{M}_{16}: 1,44, \mathrm{p}>.10\right)$.

Por último, se decidió investigar si existía alguna relación entre la capacidad de realizar gestos autodirigidos y de realizar gestos dirigidos a los otros. Con tal fin se realizó una prueba t para comparar las medias de frecuencia de los "gestos autodirigidos" entre los grupos de aquellos que realizan y aquellos que no realizan «gestos dirigidos a los otros» (gestos tipo «secuencial» y «alternado»). La comparación entre pares muestra una diferencia significativa $\left(M_{\text {dirigen a orros }}: 2,5\right.$ vs. $\mathrm{M}_{\text {no dirigen a otros: }}: 0,2, \mathrm{p}>.02$ ).

Los datos sugieren que no existen diferencias significativas entre los 13 y los 16 meses ni en la producción de gestos indicativos ni en la producción de gestos autodirigidos; y que las capacidades de autodirigirse gestos y de dirigirlos a otros se encuentran relacionadas.

\section{DISCUSIÓN}

La discusión girará en torno a cuatro ejes: el desarrollo evolutivo del gesto, la sensibilidad al contexto interpersonal, los modos de comportamiento de cada una de las tres morfologías descritas y la relación entre el señalar para sí mismo y el gesto indicativo.

1- Los análisis realizados señalan que, alrededor de los 10 meses, el gesto de señalar es poco frecuente, siendo recién alrededor de los 13 meses cuando éste parece establecerse claramente. Estos datos concuerdan con las observaciones pioneras de Schaffer (1984). Entre los 13 y los 16 meses no aparecen diferencias estadísticamente significativas en la producción del gesto de señalar, sin embargo, desde un punto de vista cualitativo, es interesante destacar que se manifiesta 
un incremento del gesto de señalar acompañado de la alternancia de la mirada hacia el objeto y la persona, mientras que decrece el uso secuencial de gesto y mirada. La alternancia entre la mirada y el gesto ha sido tradicionalmente considerada como un claro evidencia de la presencia de una intención comunicativa (Sugarman, 1984), es decir, como un indicio de que la acción es sígnica no sólo desde la perspectiva del receptor del signo sino también desde la perspectiva del niño que la produce. Ésta es una conducta que refleja la compleja comprensión que tiene el niño de los requisitos sociales necesarios para el uso del gesto; en ella se manifiesta que el niño comprende que ha de tener la atención del otro (mirada antes de realizar el gesto) para que el otro pueda seguir el gesto, y compartir con él su interés (mirada posterior al gesto) (Franco y Butterworth, 1996). En cambio, el uso secuencial de mirada y gesto no evidencia la presencia de una intención comunicativa y parece requerir una comprensión menos compleja del otro hacia quien dirige su gesto. La inversión en las frecuencia de la alternancia y secuencia de gesto y mirada permite suponer que, entre los 13 y los 16 meses, los niños se tornan más flexibles y hábiles en el uso de la mirada como recurso comunicativo.

2- Los análisis que hemos realizado muestran que los niños son altamente sensibles al contexto interpersonal en que se encuentran: las situaciones de alto y medio contacto interpersonal ("en brazos" y "al lado") son en las que más se producen gestos de señalar, siendo escasa su producción en la situación "detrás" en la que el niño se encuentra, de algún modo, solo. Esto indica que el gesto es básicamente una conducta social, una conducta que se produce principalmente cuando el niño está con otros. Nuestro diseño permite analizar de un modo más minucioso esta conducta social al relacionar las variables “situación y tipo". La sensibilidad de los niños al contexto interpersonal se manifiesta, no sólo en la variación de frecuencias en función de la situación, sino en el modo como adecuan el uso de la mirada (variable "tipo") al contexto interpersonal en el que se encuentran (variable "situación"). Cuando están en brazos la mayoría de los gestos que realizan son de tipo "solo", es decir realizan el gesto sin dirigir la mirada al adulto. Es posible que en esta situación los niños no busquen el contacto ocular, no porque su gesto no esté dirigido al otro, sino porque el estrecho contacto corporal que tienen con el otro les permite suponer que ambos están en situación de atención conjunta. La fuerte presencia del otro, a través del contacto corporal, y la casi obligada atención conjunta de hecho, dada la postura del cuerpo, no nos permiten considerar que los gestos del niño no estén dirigidos al otro (aunque nada permite negar la posibilidad de que alguno de ellos sea un gesto autodirigido). Algo cualitativamente distinto ocurre en la situación "detrás" en la que únicamente se generan gestos sin búsqueda de contacto ocular. En esta situación el contacto entre adulto y niño es casi nulo por lo que los señalamientos que el niño realiza pueden considerarse gestos autodirigidos. El análisis de la situación "detrás" nos permite afirmar que, en estados similares a la soledad, los niños, a veces, señalan. Pese a que estos datos corroboran la presencia del señalar para sí mismo, indican también la poca frecuencia con que ocurre. En la situación "al lado" se manifiestan los tres tipos de señalar, es decir el gesto sin búsqueda de contacto ocular, el gesto seguido o precedido de la búsqueda de contacto ocular y el uso alternado de gesto y mirada. En esta situación también podemos pensar que, cuando el niño señala sin buscar el contacto ocular con el adulto, está señalando para sí mismo. Un dato llama nuestra atención y es que existe un diferencia estadísticamente significativa entre el señalar para sí mismo en la situación "detrás" y en la situación "al lado". En ésta última es 
en la que el gesto ocurre más frecuentemente. Esto lleva a pensar que si bien el niño es capaz de realizar acciones semióticas autodirigidas, éstas se producen, en su mayoría, cuando se encuentra con otros. Hace ya tiempo, Vygostki (1995) mostró el error piagetiano de concebir el monólogo infantil, o habla privada, como un fenómeno no social; sus experimentos pusieron en evidencia que el habla privada se encuentra en estrecha relación con las situaciones sociales en que se produce (al poner al niño en situaciones que inhibían el habla social - compañeros de interacción sordomudos, o hablantes de distintas lenguas - el habla privada también se inhibía). Algo similar ocurre en nuestra investigación, las situaciones que favorecen la producción del gesto de señalar comunicativo son las mismas que favorecen la producción del señalar para sí mismo. Al igual que los datos obtenidos en las investigaciones de Franco, Perucchini y Butterworth (1992) y de Delgado, Gómez y Sarriá (1999) los nuestros señalan la básica condición social del señalar para sí mismo. Indican, además, que éste no precede al gesto de señalar comunicativo sino que coexiste con él. No parece que, tal como Bates (1976) sugirió, surja primero el gesto de señalar para sí mismo y que luego se lo use con fines comunicativos, sino que los niños son capaces de ser emisores y receptores de sus propios signos al mismo tiempo en que son capaces de emitir signos para otros. En los señalamientos para sí mismos que hemos observado, los niños, luego de realizarlos, no dan ninguna señal de disconformidad o displacer, por lo que debemos pensar que su función se ha satisfecho. ¿Cómo se satisface el señalar para sí mismo? De modo diferente a lo que ocurre en el señalar declarativo, en la que la función del gesto se satisface en el mirarse el uno al otro y al objeto, este modo tan peculiar de semiosis parece satisfacerse en la interacción misma del niño con el objeto. En ella la dirección de la mirada hacia el objeto, que se sostiene y se refuerza por la dirección del brazo y del dedo índice, permiten un darse cuenta del objeto de un modo, tal vez, incipientemente recursivo.

3- La interpretación de las frecuencias de los tres gestos - gesto de señalar prototípico, reaching y gesto indicativo- sólo puede hacerse a la luz de la condición general de estimulación. Como ya dijimos, ésta es semejante a la denominada por Franco y Butterworth (1996) contexto comunicativo referencial-declarativo, en el cual, mediante la presencia de un estímulo animado y distal, se busca despertar la intención del niño de compartir su interés por un objeto, sin que necesariamente se induzca el deseo de cogerlo. Casi el $80 \%$ de los gestos han sido gestos de señalar prototípicos, es decir gestos con el dedo índice extendido. El comportamiento de este gesto no presenta diferencias con el comportamiento del gesto de señalar considerado de modo global; lo dicho en los puntos anteriores, puede repetirse para el gesto de señalar prototípico. Su alta frecuencia señala que la situación declarativo referencial es claramente elicitadora de este gesto. Exactamente lo contrario ocurre con el reaching, su producción ha sido tan escasa que confirma la hipótesis de que es un gesto funcionalmente distinto al gesto de señalar y que no suele producirse en contextos declarativos. Por otro lado, la baja frecuencia del gesto indicativo confirma lo ya dicho en investigaciones previas (Lock, Young, Service y Chandler, 1990; Franco y Butterworth, 1996) acerca de la baja incidencia de este gesto. Que su frecuencia sea levemente mayor a la del reaching, gesto altamente frecuente en otras condiciones experimentales, induce a pensar que la condición experimental declarativo-referencial favorece su producción; lo cual avalaría la hipótesis de Franco y Butterworth (1996) de que éste es funcionalmente equivalente al gesto de señalar (Sin embargo, en el próximo 
punto veremos que esta equivalencia funcional puede especificarse más). Nuestros datos avalan también la hipótesis de Franco y Butterworth (1996) y de Lock, Young, Service y Chandler (1990) de que éste permanece constante en el desarrollo (al menos en el período aquí estudiado).

4- El gesto indicativo, pese a su baja frecuencia, se presenta estrechamente relacionado con el tipo "solo" de coordinación del gesto con la mirada (es decir, al realizar este gesto el niño no mira al adulto) y con las situaciones de alto y medio contacto interpersonal. Resulta evidente la similitud entre el gesto indicativo y el señalar para sí mismo, y no entre éste y el gesto de señalar en general, como se ha planteado en las investigaciones anteriores ya reseñadas: ambos son gestos que no están dirigidos a otros, a la vez que ambos se presentan con mayor frecuencia en contextos de alto y medio contacto interpersonal; lo cual indica que, pese a ser acciones semióticas autodirigidas, tienen un marcado carácter social. Su única diferencia es la morfología. Desde los trabajos de Fogel y Hannan (1985) se sabe que bebés de tres meses pueden producir una configuración semejante en su forma al gesto de señalar con el dedo índice extendido. A su vez, ya indicamos que la producción de formas similares al gesto de señalar, pero que no coinciden con la dirección de la mirada del niño (point slips out) se presenta en edades tempranas pero tiende a desaparecer alrededor de los trece meses. Éstas, como claramente señalan Lock et al. son formas silenciosas, son acciones carentes de sentido. Es decir, como esquema motor, la forma del gesto de señalar se encuentra en el repertorio de los niños desde mucho antes de que ellos lo utilicen como gesto. Estos datos y la coexistencia del gesto indicativo con el gesto de señalar nos hacen preguntar ¿por qué, si el gesto indicativo se muestra funcionalmente equivalente al señalar para sí mismo y la forma del gesto de señalar ya forma parte de sus esquemas motores, por qué — repetimos - el gesto indicativo adopta una morfología diferente? Creemos que la respuesta puede obtenerse prestando atención a su única marca diferencial: la morfología. La forma que adopta la mano en el gesto indicativo es similar a la que Povinelli y Davies (1994) describen como el patrón típico de saliencia del dedo índice cuando la mano de los humanos se encuentra en estado de distensión o relajamiento. (Patrón que se diferencia del producido por chimpancés en el mismo estado, en el cual no se presenta la saliencia del dedo índice). Es posible pensar que el gesto indicativo es un acto epistémico, un acto de contemplación del objeto, en el cual se pierde la tensión necesaria para extender el dedo índice. Ambos, el señalar para sí mismo y el gesto indicativo, parecen vincularse con el acto de tocar un objeto, acto - que como hace ya tiempo indicaron Werner y Kaplan - se encuentra ligado al mundo de los objetos contemplados, al reconocimiento de un objeto ubicado a una cierta distancia. Sólo que entre ambos existe una diferencia en el estado atencional del niño: En el gesto indicativo, el niño se encontraría en un estado distendido; en el señalar para sí mismo el niño estaría en un estado atencional similar al que debe tener para poder dirigir la atención del otro y que se refleja en la extensión del dedo índice. ¿Si la única diferencia entre el señalar para sí mismo y el gesto indicativo se debe al estado atencional en que se encuentra el niño, es posible decir que son dos gestos diferentes? No podemos aún responder esta pregunta, ella requiere nuevas investigaciones, pero sí se desprende de nuestro trabajo que, desde edades tempranas, los niños realizan gestos de señalar, que pese a no estar dirigidos a los otros, tienen una naturaleza social; que en el desarrollo humano, al menos en los intervalos que nosotros hemos estudiado, la capacidad de dirigir signos hacia los otros y de dirigirlos a sí mismo surgen a la vez; y que los niños que son capaces de autodirigirse signos son también capaces de dirigirlos a los otros. 


\section{Notas}

${ }^{1}$ Un cuarto grupo estaría formado por la propuesta reciente de Butterworth (1998) en el que se apela al proceso de antítesis darwiniano para explicar el origen del gesto.

Se van a tener en cuenta los efectos con probabilidad menor de .10 dado que se presentan siempre las $\mathrm{F}$ corregidas con el método Greenhouse cuando no se ha cumplido el supuesto de esfericidad. Los grados de libertad que se presentan son los originales.

3 Las comparaciones entre pares de medidas se hicieron mediante prueba t corregida por el método de Bonferroni.

\section{Referencias}

BAtes, E. (1976). Language and Context: The acquisition of pragmatics. Nueva York: Academic Press.

Bates, E., Benigni, I., Bretherton, L., Camaioni, L. y Volterra, V. (1979). Cognition and communication for nine to thirteen months: Correlational findings. En E. Bates (Ed.), The emergence of symbols: Cognition and communication in infancy. Nueva York: Academic Press.

Bates, E., Camaioni, L. y Volterra, V. (1975). The acquisition of performatives prior to speech. Merrill-Palmer Quarterly, 21, 205-226.

Butterworth, G. (1998). Origins of social visual attention in infancy. Monographs of the society for research in child development, 225, 63 (4), 144-166.

Camaioni, L., Perucchini, P., Murator, F. y Milone, M. (1997). Brief report: A longitudinal examination of the communicative gestures deficit in young children with Autism. Journal of Autism and Developmental Disorders, 27 (6), 715-725.

Carpenter, M., Nagell, K. y Tomasello, M. (1998). Social cognition, joint attention, and communicative competence from 9 to 15 months of age. Monographs of the society for research in child development, 225, 63 (4).

Delgado, B., Gómez, J. C. y SARriá, E. (1999). Non communicative pointing in preverbal children. Poster presentado en IXth European Conference on Developmental Psychology, Isla de Spetses.

D'odorico, L. y Levorato, C. (1990). Social and cognitive determinants of mutual gaze between mother and infant. En V. Volterra y C. J. Ertings (Eds.), From gesture to language in hearing and deaf children. Berlín: Springer-Ver lag.

ESPAÑOL, S. (1999). The emergence of the pointing gesture: between communicating and sharing. Comunicación presentada en Sixth International Colloquium on Cognitive Science, San Sebastián.

Fogel, A. y Hannan, T. E. (1985). Manual actions of 2 to 3 month-old human infants during social interaction. Child Development, 56, 1271-79.

Franco, F., Perucchini, P. y Butter worth, G. (1992). Pointing for an agemate in 1-2 years olds. Poster presentado en Fifth European Conference of Developmental Psychology, Sevilla.

Franco, F. y Butterworth, G. (1996). Pointing and social awarness: declaring and requesting in the second year. Journal of Child Language, 23, 307-336.

Gómez, J. C., SARrí́, E. y TAMARIt, J. (1993). “The comparative study of early communication and theories of mind: ontogeny, phylogeny and pathology". En S. Baron-Cohen, H. Tager-Flusberg y D. Cohen (Eds.), Understanding other minds: perspective from autism. Oxford: Oxford University Press.

GómeZ, J. C. y NúÑEZ, M. (1998). La mente social y la mente física: desarrollo y dominios de conocimiento. Infancia y Aprendizaje, 84, 5-32.

Goodhart, F. y BARon-Cohen, S. (1998). How many ways can the point be made? Evidence from children with and without autism. First Language, 13, 225-233.

KAYE, K. (1982). The Mental and social life of the babies. Londres: Methuen.

León, O. y Montero, I. (1997). Diseño de Investigaciones. Madrid: McGraw-Hill.

Leung, E. y Rheingold, H. (1981). The development of pointing as a social gesture. Development Psychology, 17, 215-220.

LOCK, A. (1980). The guided reinvention of language. Londres: Academic Press.

Lock, A., Young, A., Service, V. y Chandler, P. (1990). Some observation s on the origin of the pointing gesture. En V. Volterra y C. J. Erting (Eds.), From gesture to language in hearing and deaf children. Berlín: Springer-Verlag.

Morissette, P., Ricard, R. y Gouin Dècarie, T. (1995). Joint visual attention in infancy: A longitudinal study of comprehension. British Journal of Developmental Psychology, 13 (2), 163-177.

Murphy, C. (1978). Pointing in the context of a shared activity. Child Development, 49, 371-380.

Murphy, C. y Messer, D (1977). Mothers, infants and pointing: a study of gesture. En R. H. Schaffer (Ed.), Studies in mother-infant interaction. Londres: Academic Press.

Perucchini, P. (1997). Sviluppo delle funzioni richiestiva e dichiarativa del gesto di indicare. Giornale italiano di psicologia, 24 (4), 813-829. 


\section{5}

Perucchini, P. y Camaioni, L. (1993). When intentional communication emerges? Developmental dissociations between declarative and imperative functions of the pointing gesture. Poster presentado en The BPS Annual Conference, Developmental Section, Birmingham.

Phillips, W., Gómez, J.C., Baron-Cohen, S., LaA, V. y Rivière, A. (1995). Treating people as objects, agents, or "subjects": How young children with and without autism make requests. Journal of Child Psychology and Psychiatry and Allied Disciplines, 36 (8), 1383-1398.

Povinelli, D.J. y Davis, D. R. (1994). Differences between chimpanzees (Pan troglodytes) and humans (Homo sapiens) in the resting state of the index finger: implications for pointing. Journal of Comparative Psychology, 108 (2), 134-139.

RivièRe, A. (1997). Teoría della metarappresent azione. En F. Braga Illa (Ed.), Livelli di rappasentazione. Urbino: Quatro Venti.

Rivière, A. y Sotillo, M. (1999). Comunicazione, sospensione e semiosi umana: le origini della pratica e della compresione interperson ali. Ricerche di sociologia epsicologia della comunicazione, 1, 45-76.

SARriá, E. y Rivière, A. (1991). Desarrollo cognitivo y comunicación intencional preverbal: un estudio longitudinal multivariado. Estudios de Psicología, 46, 35-52.

SCAIfE, M. y BRUner, J. (1975). The capacity for joint visual attention in the infant. Nature, 25, 265266.

SCHAFFER, H. R. (1984). The child's entry into a social world. Londres: Academic Press.

Sugarman, S. (1984). The development of preverbal communication: its contribution and limits in promoting the development of language. En R. Schiefelbusch y Pickar (Eds.), The acquisition of communicative competence. Baltimore: University Park Press.

Trevarthen, C. y Hubley, P. (1978). Secondary intersubjectivity: confidence, confiding and acts of meaning in the first year. En A. Lock (Ed.), Action, gesture and symbol: the emergence of language. Londres: Academic Press.

VyGOTSKI, L. S. (1995). Pensamiento y lenguaje. Barcelona: Paidós.

Werner, H. y Kaplan, B. (1984). Symbol formation. Hillsdale, NJ: Lawrence Erlbaun Associates Inc. 